\title{
Niveles de ansiedad en nińos victimizados sexualmente que deben declarar en juicios orales: aportes de un programa de preparación
}

\author{
Anxiety levels in victimized children who must testify in \\ oral trials: contributions of a training program \\ Cristóbal Guerra Vio ${ }^{1}$ \\ Marcela Viveros Barrera ${ }^{2}$ \\ Blanca Calvo Lazo ${ }^{3}$ \\ Pamela Canessa Quiroz ${ }^{4}$ \\ Franco Mascayano Tapia ${ }^{5}$
}

\section{Resumen}

Existe evidencia de que niños víctimas de delitos sexuales que enfrentan procesos judiciales pueden sufrir victimización secundaria. Diversos autores sugieren preparar a los niños para enfrentar dicha instancia y disminuir los riesgos de victimización secundaria. Este artículo describe los niveles de ansiedad de ocho nińos expuestos a un programa de preparación para nińos victimizados sexualmente que deben declarar en juicios. Se evaluó su nivel de ansiedad antes e inmediatamente después del juicio. No se observaron diferencias significativas entre ambas mediciones. Pese a los resultados, se considera necesario que futuras investigaciones evalúen de forma rigurosa el programa y su capacidad de prevención de la victimización secundaria. Finalmente, se discuten las implicancias de este tipo de programas en el sistema judicial chileno.

Palabras clave: abuso sexual infantil, victimización secundaria, juicio oral, programa de preparación.

\footnotetext{
1 Psicólogo, Magíster en Psicología. Docente de la Escuela de Psicología de la Universidad Santo Tomás. E- mail: cguerravio@yahoo.es

2 Psicóloga del Centro de Atención a Víctimas de Delitos Violentos de la Corporación de Asistencia Judicial de Valparaíso. E- mail: marcelaviveros@hotmail.com

3 Abogada del Centro de Atención a Víctimas de Delitos Violentos de la Corporación de Asistencia Judicial de Valparaíso. E- mail: blanca_calvo_laso@hotmail.com

4 Abogada, Magíster en Derecho Penal. Abogada del Centro de Atención a Víctimas de Delitos Violentos de la Corporación de Asistencia Judicial de Valparaíso. E- mail: pamelacanessa@gmail.com

5 Psicólogo de la Universidad de Valparaíso. E- mail: franco.mascayano@gmail.com
} 


\begin{abstract}
There is evidence that child victims of sexual crimes, who are facing legal proceedings, may suffer secondary victimization. Several authors suggest preparing children to face this request and thus reduce the risk of secondary victimization. This article aims to describe the Anxiety levels in eight children who were trained in a program for sexually victimized children who need to testify in oral trial. We evaluated anxiety level before and immediately after the trial. There were no significant differences between the two measurements. Despite the favorable results, we consider further investigation necessary in order to evaluate program effectiveness and its ability to prevent secondary victimization. Finally, we discuss the implications of such programs in the Chilean judicial system.
\end{abstract}

Key words: child sexual abuse, secondary victimization, oral judgment, training program.

\title{
Introducción
}

Cuando un niño declara haber sido víctima de un delito sexual, y esto se formaliza en una denuncia ante la fiscalía, se inicia un proceso judicial tendiente a esclarecer la situación. Este proceso judicial incorpora una serie de etapas, entre las que se cuentan varias declaraciones que tiene que realizar el niño víctima en la fase de investigación (ante la policía, el fiscal, el perito médico, el perito de credibilidad, etc.) y, de encontrarse antecedentes suficientes, debe repetir la declaración ante el tribunal oral y los respectivos abogados (fiscal, defensor y eventualmente un abogado querellante). Para muchos autores este proceso judicial deviene en un fenómeno altamente ansiógeno denominado victimización secundaria.

Echeburúa, De Corral y Amor (2002) convienen en que la victimización primaria se relaciona directamente con los hechos delictivos y sus consecuencias a corto plazo. En cambio, señalan que la secundaria, se establece en función de la relación entre la víctima y el sistema jurídicopenal. Gutiérrez, Coronel y Pérez (2009) apuntan a que la victimización secundaria genera una serie de consecuencias negativas a nivel psicológico, 
social, jurídico y económico producto de las diferencias entre las legítimas expectativas de la víctima y la realidad institucional jurídica. Rubio y Monteros (2001) agregan que las consecuencias de la victimización secundaria pueden ser tan graves como las de la victimización primaria. Específicamente, se ha descrito que la victimización secundaria producida por las continuas declaraciones en el sistema penal se asocia a altos niveles de ansiedad en la víctima (Gutiérrez et al., 2009).

Uno de los agentes más influyentes en la génesis de la victimización secundaria atañe principalmente a la actuación de la policía y del sistema judicial (jueces, forenses, fiscales y abogados). Es muy frecuente que dichos personeros se interesen principalmente en los procesos burocráticos ligados a la investigación (búsqueda de pruebas), descuidando el trato dado a la víctima (Echeburúa \& Subijana, 2008). Esta característica del sistema legal da cuenta de una falta de consideración por el profundo impacto psicosocial que los abusos sexuales producen en las personas en general, $\mathrm{y}$ más aún, en población infantil (Álvarez \& Smith, 2007).

En esta línea, diversos autores han enunciado los principales factores que inciden en el desarrollo del fenómeno de victimización secundaria. Destacan entre ellos la escasez de información entregada a la víctima sobre las características del proceso penal, la sobreestimación de la investigación pericial (en desmedro de la atención integral de la víctima), la excesiva lentitud de los juicios (que interfiere directamente con los procesos de readaptación de las personas violentadas) y los elementos propios del juicio oral (narración del delito en presencia del victimario, cuestionamiento directo acerca de la credibilidad de la víctima, generación de sentimientos de culpabilidad, vergüenza y altos niveles de ansiedad, entre otros) (Álvarez \& Smith, 2007; Arce \& Batres, 2006; Gutiérrez et al., 2009; Lewis, 2003; Reno et al., 1999).

Hernández y Miranda (2005) sostienen que la declaración de los niños en juicios orales resulta uno de los desafíos más importantes para el sistema penal. Estos autores señalan que la inclusión de la víctima en los procesos legales juega un rol esencial en la resolución del juicio, y a su vez, en su propio proceso de reparación. Por ende, es atingente la integración de la víctima por parte del sistema legal, dotándola de un estatuto 
jurídico específico, cimentado en cuatro grandes directrices: información, participación, asistencia y protección (Celia, 2008). Resulta trascendente complementar el marco judicial, dedicado a la investigación de los eventos y las posibles penas con respecto al agresor, con el marco terapéutico, abocado a la asistencia integral del niño. Cada marco debería confluir sinérgicamente, unidos en la persecución de un fin último: la protección del niño. Es deber de los profesionales encargados de la asistencia terapéutica entregar información detallada de las competencias comunicacionales del niño, puntualizando las posibles secuelas psicológicas que podrían desencadenarse a raíz de su declaración en un juicio oral. Por su parte, el área judicial debería velar porque la intervención del niño en el juicio se limite a las interacciones realmente necesarias, para evitar el sufrimiento por parte del niño, que se sume al ya vivenciado con el hecho delictual (Echeburúa \& Subijana, 2008).

La "Convención de Derechos de los Niños" (Unicef, 1989), propone directrices para velar por los intereses de los niños en los procesos judiciales. Entre estas directrices destacan las referidas a la necesidad de crear conciencia acerca del impacto de la participación de los niños víctimas y testigos en los mecanismos de justicia, a la necesidad de reconocer los factores vinculados al proceso legal que influyen en el bienestar del niño, además de potenciar un testimonio de calidad. Por otro lado, se plantea la necesidad de considerar los derechos de los niños testigos y víctimas, para de ese modo brindarles el apoyo necesario para enfrentar de mejor manera los procedimientos propios del sistema penal (ej. el uso de videoconferencias, uso de seudónimos, mantenimiento del anonimato de los niños, etc.) (Unicef, 2007).

Algunos autores señalan que es necesario que existan programas específicos de apoyo a niños que declaren en juicios, que incluyan el acompañamiento del niño para conocer la sede judicial donde deberá declarar y la preparación de la declaración en el juicio oral (Echeburúa \& Subijana, 2008; Reno et al., 1999). Lipovsky (1997), agrega que este trabajo debe realizarse por un equipo multidisciplinario que incorpore profesionales del ámbito legal y social. Michels (2009), afirma que la disponibilidad de apoyo psicosocial, en combinación con las estrategias 
de protección eficaces, potencian la participación segura del niño en el proceso judicial, mejorando la calidad de su testimonio y protegiéndolo de posibles daños derivados de la interacción en juicio oral. El autor señala que el asesoramiento individual ofrecido a un niño, antes que este testifique, permite al profesional de apoyo identificar las necesidades, deseos y capacidades de este niño en relación al juicio. De este modo el profesional puede construir una relación de confianza que le permita preparar al niño para el proceso de dar testimonio y así otorgarle una sensación de control sobre el mismo (de forma que disminuya la ansiedad en el niño y se prevenga la victimización secundaria).

Plotnikoff y Woolfson (2001), plantean recomendaciones más específicas que han sido plasmadas en un programa de preparación a niños para enfrentar instancias judiciales. Estos autores proponen diferentes momentos en la preparación de la testificación por parte de las víctimas; señalan que, en etapas iniciales, es prioridad acompañar a la víctima en las primeras declaraciones. Agregan que, en fases posteriores, juega un importante rol la entrega de información al niño acerca de su testificación en el sistema legal y la realización de ejercicios que le permitan a este habituarse a los estímulos que estarán presentes en el juicio. Finalmente, indican que es prioritario evaluar las competencias comunicacionales y de afrontamiento a situaciones estresantes que poseen los nińos, para así planificar entrenamientos específicos en dichas áreas, claves para un desempeño apropiado en juicios orales. Los mismos autores revelan los resultados de la evaluación del programa en víctimas que testificaron en procesos penales en Escocia. Participaron de esa investigación 151 niños (de los cuales 110 participaron del programa y 41 no lo hicieron), de edades entre 5 y 17 ańos. Mediante una entrevista semiestructurada se evaluó la percepción de los niños respecto a la capacitación entregada. El apoyo proporcionado por los sistemas de especialistas fue bien recibido por los niños testigos. Noventa y seis por ciento de los testigos que recibieron el adiestramiento comentaron una apreciación de confianza en el Defensor, facilitando el hecho de acudir a los tribunales. El grupo de niños que participaron del programa de soporte psicosocial reportó niveles significativamente más altos de apoyo percibido que el grupo comparación. 
Además, los niños que participaron del programa señalaron que estuvieron mejor informados sobre qué y cómo responder en la corte, y lo que puede ocurrir durante el interrogatorio, que los del grupo de control (Plotnikoff $\&$ Woolfson, 2007).

En España, Alarcón et al. (2008) proponen un programa de características similares al recién descrito. Los propósitos del programa son, por un lado, mejorar la declaración del testigo-víctima (aumentar su credibilidad) y, por otro, garantizar los derechos de la víctima (evitar la victimización secundaria, brindar contención emocional a la víctima y reducir el estrés). El programa ha sido aplicado a más de 1245 sujetos, con resultados auspiciosos, según lo mencionado por los mismos autores.

En Chile, Canessa y Guerra (2010) diseñan un programa de preparación a niños víctimas de delitos sexuales que deben declarar en juicios orales. El programa de entrenamiento pretende favorecer la transformación de la instancia de juicio oral en una instancia de reparación para el niño, evitando que esta instancia se constituya en una fuente de victimización secundaria generadora de ansiedad. El programa, que integra una serie de estrategias cognitivo conductuales en diez fases, fue diseñado para ejecutarse en un período máximo de cuatro semanas. La primera fase se denomina "fase social" ya que tiene por objetivo que el niño conozca a quienes realizarán el entrenamiento (habitualmente un psicólogo y un abogado), sin abordar los temas asociados al abuso. La segunda fase de "evaluación" busca conocer las emociones y creencias que el niño tiene acerca del juicio. Luego en la etapa de "explicación del juicio oral" se realiza un proceso de psicoeducación en el que, utilizando apoyos didácticos, se explica al niño la dinámica del juicio. En la cuarta etapa de "visita al tribunal oral" se acude a una sala vacía donde se realizan juicios orales, con el objeto de que el niño se familiarice con el lugar en que prestará la declaración. En la quinta etapa de "manejo de la ansiedad" se enseñan al niño estrategias de respiración, relajación y autoinstrucciones, posibles de ser implementadas antes y durante el juicio. Luego, en la sexta etapa se realiza una "simulación del interrogatorio" mediante juego de roles. En la séptima etapa de "reforzamiento positivo" se destacan las características positivas del niño de forma 
que aumente su sensación de autoeficacia. En la octava etapa se realiza una "entrevista con los padres" (o adultos responsables), para orientarlos en la forma de acoger al niño antes y después del juicio oral. Luego de estas ocho etapas, el niño declara en el juicio oral. Inmediatamente después de ello se realiza la novena etapa denominada "entrevista contingente a la finalización del juicio", donde se acoge y refuerza al niño por su desempeño en el juicio. Esta entrevista es breve (un par de minutos) y puede realizarse personalmente o por teléfono. La última etapa, de "cierre", se realiza máximo una semana después de finalizado el juicio. En ella se entregan los resultados del juicio e, independiente de ellos, se refuerza al niño por su desempeño.

El programa no ha sido evaluado formalmente, solo se cuenta con el reporte de dos niños varones (de 5 y 8 años, respectivamente) que fueron entrenados según el procedimiento descrito. Ambos niños, luego de declarar en un juicio, realizan una evaluación favorable del proceso judicial y de su propio desempeño en él (Canessa \& Guerra, 2010).

Como puede apreciarse en los párrafos precedentes, existen numerosos autores que apuntan a la necesidad de generar programas de apoyo a niños que deben declarar en juicios orales. No obstante, son escasas las propuestas concretas referidas a la forma de implementar estos programas y escasa es también la evidencia de su efectividad.

El presente estudio pretende describir los niveles de ansiedad de una muestra de niños, participantes del programa de Canessa y Guerra (2010), en situaciones de pre y post declaración en juicio oral. Adicionalmente se pretende analizar las implicancias que pudiera tener, en el sistema penal chileno, la aplicación de este tipo de programas de preparación a víctimas.

Método

Participantes

Participaron 8 niños ( 1 de género femenino y 7 de género masculino) víctimas de delitos sexuales reiterados a nivel intrafamiliar. La edad de los nińos fluctuó entre los 8 y los 13 ańos $(M=10,13$; $\mathrm{DT}=1,55)$. Todos 
ellos estaban siendo atendidos en un Centro de Atención Integral a Víctimas de Delitos Violentos de la Corporación de Asistencia Judicial de la Región de Valparaíso (CAVI). Se trabajó con todos los niños atendidos en dicho centro que debieron declarar en juicios orales durante el año 2010. Dentro de este centro los nińos comenzaron a recibir atención psicológica entre dos y tres meses después de la realización de la denuncia en el Ministerio Público. Al momento de realizar la investigación los niños se encontraban asistiendo a psicoterapia individual por un período de entre 4 y 6 meses $(M=4,75$; DT $=$ 0,71). Los niños fueron atendidos por dos psicólogos diferentes (un psicólogo y una psicóloga). Pese a que al inicio de la terapia fueron evaluados con procedimientos distintos, en la totalidad de los casos existía sintomatología ansiosa y depresiva asociada a los hechos abusivos. Todos los niños contaron con apoyo de figuras adultas significativas que cooperaron con el proceso terapéutico y con el proceso judicial. El detalle de las características demográficas de cada niño puede apreciarse en la Tabla 1.

\section{Instrumento}

Dado que interesaba conocer el nivel de ansiedad de los niños en momentos específicos (antes e inmediatamente después de la declaración en el juicio oral), se utilizó la subescala "ansiedad estado" de la adaptación española de la escala de ansiedad estado-rasgo (STAIC) (Seisdedos, 1990). La STAIC es una escala de autorreporte de 20 reactivos que evalúa el nivel de ansiedad del niño en un momento determinado. La escala arroja un puntaje global de ansiedad estado. Para obtener dicho puntaje se deben invertir los valores de algunos ítems (que miden la ansiedad inversamente) y luego sumar los valores de los 20 ítems de la escala. Los puntajes oscilan entre 20 y 60. A mayor puntaje mayor es la ansiedad estado del nińo.

Para favorecer la comprensión de los reactivos por parte de nińos chilenos se realizaron pequeños cambios en tres reactivos. En el ítem 19 se cambió la palabra "contrariado" por "enojado", en el ítem 13 se cambió la palabra "agradable" por "cómodo" y en el ítem 15 se cambió "confuso" por "confundido". 


\section{Procedimiento}

Tres semanas antes del juicio oral se inició el proceso de preparación de los niños para enfrentar dicha instancia, según el protocolo de Canessa y Guerra (2010). El programa de preparación fue implementado por el psicólogo o psicóloga tratante del niño y por una abogada. Los padres (o adultos responsables) de estos nińos dieron su consentimiento para que estos participen del programa de preparación.

Las fases 1 a 3 (fase social, de evaluación y de explicación del juicio oral) se realizaron en dependencias del CAVI, en dos sesiones, durante la primera semana de entrenamiento. Las fases 4 y 5 (visita al tribunal oral y de manejo de la ansiedad) se realizaron, en dos sesiones, durante la segunda semana. La visita al tribunal se realizó en una sala de audiencia de un tribunal oral en lo penal de la Región de Valparaíso, mientras que la fase de manejo de la ansiedad se realizó en las dependencias del CAVI. Las fases 6,7 y 8 (simulación del interrogatorio, de reforzamiento positivo y entrevista con los padres) se realizaron, en tres sesiones, durante la tercera semana de entrenamiento (dos sesiones con el nińo y una con los padres o adultos responsables). Todas ellas fueron realizadas en las dependencias del CAVI. En la fase 7 (de reforzamiento positivo) se aplicó a los niños la STAIC (aplicación pre). Se optó por realizar la aplicación inicial de la escala en la fase 7 debido a que esta es la última fase de preparación con el niño antes del juicio, por lo que se estimó que en ese momento los niños ya se encontraban habituados a la situación de juicio y tenían las herramientas para controlar el estrés. Además la fase 7 es una fase donde se destacan los recursos del niño sin exponerlo a situaciones ansiógenas. De este modo resulta interesante comparar la ansiedad en una situación amigable con la ansiedad manifestada posteriormente por los niños en una situación más estresante (juicio). Luego de las ocho etapas iniciales cada niño debió declarar en su respectivo juicio oral. Inmediatamente después de cada declaración, y en el mismo tribunal, se aplicó nuevamente la STAIC (aplicación post) y se realizó la novena fase (entrevista contingente a la finalización del juicio). La fase de cierre se realizó en el CAVI una semana después de terminado el juicio. 
Hay que señalar que inicialmente se pensó en formar un grupo experimental con los niños usuarios de centros reparatorios y un grupo control con aquellos que no lo son (pero que igualmente deben declarar en juicios orales, aunque sin ningún tipo de preparación). Para ello se solicitó apoyo a un tribunal oral en lo penal. El apoyo solicitado fue para aplicar el instrumento al eventual grupo control. El tribunal se negó aludiendo a dos razones. Por un lado señaló que este apoyo podría ser interpretado por la defensoría como una actitud "pro-víctima" que reste neutralidad, objetividad y credibilidad al tribunal. Por otro lado cuestionó el protocolo mismo, señalando que el que los niños enfrenten el juicio oral con menos ansiedad les restaría credibilidad en su declaración. Ante esta negativa quedaba la posibilidad de trabajar exclusivamente con niños que asisten a centros reparatorios y preparar a algunos de ellos (para que constituyan el grupo experimental) y no preparar a otros (para que constituyan el grupo control). No obstante, por razones éticas (Colegio de Psicólogos de Chile, 1999), se consideró poco recomendable privar a algunos de estos niños de un protocolo potencialmente beneficioso para ellos. Este tema será ahondado en la sección discusión.

\section{Resultados}

Se comparó el nivel de ansiedad que experimentaron los niños una semana antes del juicio (en una situación amigable) con el nivel de ansiedad experimentado inmediatamente después de finalizado el juicio. A continuación se ofrece un análisis individual de los resultados obtenidos por cada uno de los niños y luego un análisis grupal que contempla los valores promedio obtenidos por los niños.

La Tabla 1 muestra los niveles de ansiedad previa y posterior al juicio de cada uno de los ocho niños que participaron de este estudio. La ansiedad de estos fue medida con la STAIC, que ofrece valores posibles entre 20 y 60 puntos (a mayor puntaje mayor es el nivel de ansiedad estado).

Se observa que previo al juicio los niños presentaron bajos niveles de ansiedad (puntajes en la STAIC entre 20 y 29 puntos). Posterior al 
juicio los niveles de ansiedad, aún siendo bajos, presentaron una mayor oscilación (puntajes en la STAIC entre 20 y 33 puntos). Además se observa que los niveles de ansiedad de los niños aumentaron levemente en seis casos y disminuyeron levemente en los otros dos.

Tabla 1

Caracteristicas demográficas y niveles de ansiedad (puntajes STAIC previos y posteriores al juicio oral) de los participantes del estudio

\begin{tabular}{ccccc}
\hline Género & $\begin{array}{c}\text { Edad } \\
\text { (años })\end{array}$ & $\begin{array}{c}\text { Tiempo en } \\
\text { terapia } \\
\text { (meses })\end{array}$ & $\begin{array}{c}\text { Ansiedad } \\
\text { previa al } \\
\text { juicio }\end{array}$ & $\begin{array}{c}\text { Ansiedad } \\
\text { inmediatamente } \\
\text { después de } \\
\text { declaración }\end{array}$ \\
\hline Niño & 8 & 4 & 21 & 24 \\
Niña & 9 & 5 & 22 & 20 \\
Niño & 10 & 5 & 20 & 25 \\
Niño & 10 & 6 & 25 & 26 \\
Niño & 9 & 5 & 23 & 23 \\
Niño & 11 & 4 & 29 & 33 \\
Niño & 13 & 5 & 26 & 33 \\
Niño & 11 & 4 & 29 & 26 \\
\hline
\end{tabular}

Por su parte, la figura 1 muestra los valores promedio de ansiedad obtenidos por los niños en ambas mediciones. En la medición previa al juicio los niños obtuvieron, en promedio 24,38 puntos (DT=3,46) en la STAIC, mientras que en la medición posterior al juicio el nivel de ansiedad alcanzó los 25,63 puntos (DT= 4,10). Para evaluar si las diferencias entre la aplicación previa y posterior al juicio son o no estadísticamente significativas se realizó un análisis con la prueba no paramétrica $\mathrm{z}$ de Wilcoxon. Este análisis indicó que los niveles de ansiedad experimentados por los niños posterior al juicio no se diferencian significativamente de los experimentados una semana antes $(\mathrm{z}=-0,98 ; \mathrm{p}=0,33)$. 
Figura 1

Puntajes promedio de ansiedad previa y posterior al juicio, según la STAIC $(n=8)$

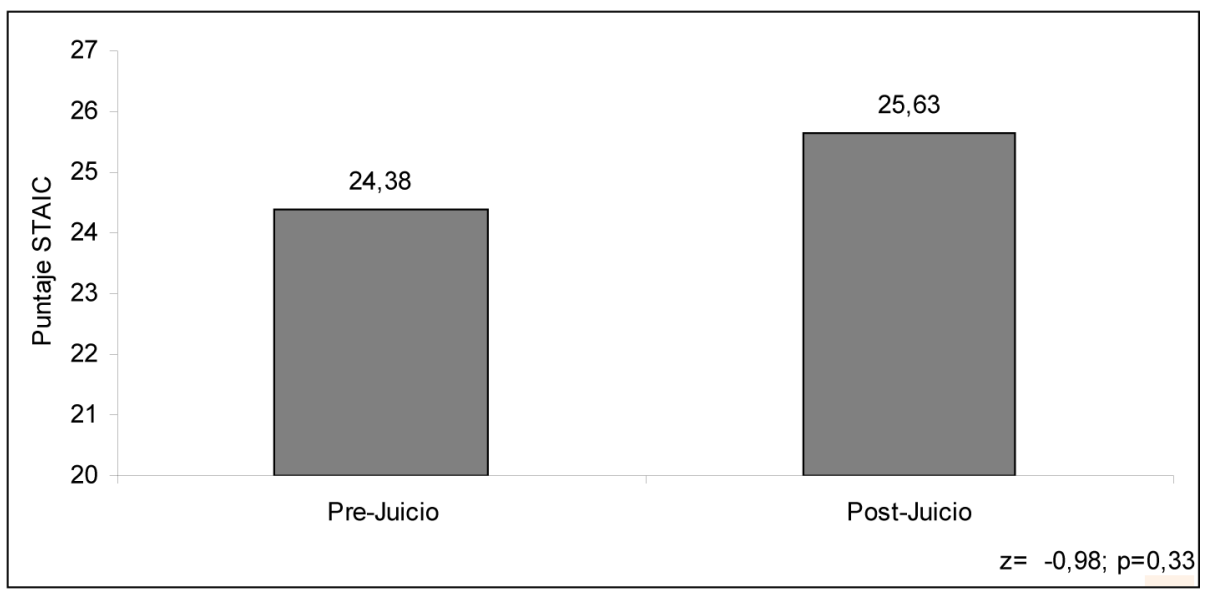

Discusión

El primer objetivo del presente estudio fue describir los niveles de ansiedad de los niños que participaron del programa de Canessa y Guerra (2010), en situaciones de pre y post declaración en un juicio oral. Los resultados indican que los niveles de ansiedad experimentados por los niños preparados con este protocolo no presentan variaciones estadísticamente significativas al comparar los niveles previos a la declaración con los presentados inmediatamente después de esta. Estos resultados son coherentes con el planteamiento de diversos autores respecto de la necesidad de preparar la declaración de los niños de forma de prevenir la victimización secundaria (Echeburúa \& Subijana, 2008; Hernández \& Miranda, 2005; Lipovsky, 1997; Michels, 2009; Reno et al., 1999).

Pese a lo auspicioso de los resultados, este estudio pre experimental presenta varias limitaciones que hacen que sus resultados deban ser considerados con cuidado. En este sentido, es necesario resaltar que solo es posible concluir que los niños entrenados presentaron bajos niveles de ansiedad estado pre y post declaración, pero no se puede señalar que esto dé cuenta de la efectividad del programa de preparación. Lo anterior se 
sustenta en varios argumentos: primero, al medir la ansiedad estado en la fase siete del programa de preparación es altamente probable que los bajos niveles de ansiedad de los niños ya estén influidos por el programa recibido hasta ese momento; segundo, debido a que no se incluyó un grupo de comparación, el control experimental fue escaso; tercero, al trabajar con una muestra pequeña, los resultados no pueden ser generalizables al resto de la población.

Se considera que, aun siendo un estudio acotado, los resultados obtenidos abren una línea de investigación en Chile que aporta a la escasa evidencia empírica existente en la actualidad sobre los efectos de programas de apoyo a víctimas que deben declarar en el proceso penal. Hay que recordar que, en la revisión bibliográfica asociada al presente artículo, solo se accedió a dos estudios extranjeros que se refieren a los efectos de este tipo de programas (Alarcón et al., 2008; Plotnikoff \& Woolfson, 2007).

Es necesario que a futuro se realicen estudios tendientes a evaluar la efectividad del programa de entrenamiento de Canessa y Guerra (2010) de forma concluyente. Para ello se debe trabajar con una muestra más representativa y con mayor control experimental. Específicamente, se sugiere incluir un grupo de control, evaluar la ansiedad de los niños antes de iniciar el entrenamiento y controlar posibles variables extrañas que pueden incidir en los niveles de ansiedad experimentados por estos. Entre las posibles variables extrañas habrá que poner especial atención en: el nivel de ansiedad rasgo de los niños, que explique el manejo individual de la ansiedad característico de cada uno de ellos; su género, pues en este estudio se trabajó principalmente con varones; y a las características de la terapia reparatoria que los niños han recibido previamente y que pueden haber dotado a los niños de estrategias de manejo de la ansiedad no incluidas en el programa.

Por otro lado, el segundo objetivo de este artículo fue analizar las implicancias de la aplicación de este tipo de programa en Chile. En este sentido, es necesario retomar lo señalado en el apartado "procedimiento" respecto al comentario emitido por personeros de un tribunal oral al negarse a cooperar con el estudio. Por un lado, señalaron que el apoyo 
a este tipo de estudios podría ser interpretado como una actitud a favor de la víctima (y en desmedro del imputado) y, por otro, se cuestionó la utilidad jurídica de que los niños enfrenten el juicio oral con menores niveles de ansiedad (ya que parecerían menos creíbles). Esta situación abre el viejo debate sobre el objetivo de que los niños victimizados sexualmente declaren en juicios orales.

Barrera y Guerra (2007) dan cuenta de que los representantes del sistema jurídico tienen diferentes miradas respecto de la infancia y respecto del abuso sexual infantil que los profesionales de los equipos de reparación. Estos autores señalan que para el sistema jurídico la víctima es considerada un "objeto de derecho", que debe cooperar con la justicia para el esclarecimiento de los hechos delictivos. En cambio, para los profesionales de los equipos reparatorios la víctima debe ser considerada un "sujeto de derechos" que debe beneficiarse del sistema judicial, para el logro de la reparación. En este sentido la negativa de los tribunales para cooperar en este estudio es altamente coherente con la visión de la víctima como "objeto de derecho". Además es contraria a los planteamientos de la "Convención de Derechos de los Niños" (Unicef, 1989), que sugieren una consideración especial por los niños que participan en instancias judiciales, y contraria también a la contundente evidencia respecto del papel que tienen las instancias jurídicas en el surgimiento de la victimización secundaria (Álvarez \& Smith, 2007; Arce \& Batres, 2006; Gutiérrez et al., 2009; Echeburúa \& Subijana, 2008; Lewis, 2003; Reno et al., 1999). De este modo, se considera que, dado que Chile está adscrito a la Convención Internacional de Derechos del Niño (Unicef, 1989) se debiera velar porque todas las instituciones públicas o privadas de bienestar social, los tribunales, las autoridades administrativas, y los órganos legislativos, consideren de manera explícita el interés superior del niño. No se trata de tener una "actitud pro-víctima" que sea usada en desmedro de los derechos del imputado, sino de considerar que esa víctima es un niño que merece ser tratado con una consideración especial, producto de su calidad de niño. Tampoco se trata de utilizar este tipo de protocolo para inducir la declaración del niño, sino de prepararlo emocionalmente para que no sufra una victimización secundaria. 
Es importante que los programas de preparación a niños victimizados sexualmente que deben declarar en juicios orales sean coherentes con la legislación del país en el que deberán ser aplicados. De aquí que resulta de utilidad contar con programas de apoyo a las víctimas diseñados específicamente para ser usados en Chile.

Finalmente, se considera que quien aplique este tipo de programa debe ser consciente de las resistencias que puede generar en el sistema penal (dadas las diferentes concepciones de víctima que hay entre los profesionales encargados de la reparación de los nińos victimizados y el sistema legal).

\section{Referencias}

Alarcón, L., Aragonés, R., Bassa, M., Farrán, M., Guilén, J., Juncosa, X., et al. (2008). Comunicación sobre el Programa de Apoyo a la Exploración Judicial de Testigos Vulnerables en Cataluña. Anuario de Psicología Jurídica, 18, 11-20.

Álvarez, M. \& Smith, B. (2007). Revictimización: Un fenómeno invisibilizado en la instituciones. Revista Medicina Legal de Costa Rica. 24(1), 65-101.

Arce, A. \& Batres, J. (2006). Una aproximación a los efectos psicosociales, producto de la victimización secundaria en niños y niñas víctimas de los delitos contra la libertad y seguridad sexuales que asistieron al ministerio público. Tesis para optar al grado de Licenciado en Psicología no publicada, Universidad San Carlos de Guatemala.

Barrera, P. \& Guerra, C. (2007) Creencias de profesionales que atienden a víctimas de abuso sexual infantil respecto de la influencia del proceso legal en su desgaste y satisfacción laboral. En Corporación de Asistencia Judicial (Ed.) Atención a Victimas de Delitos Violentos: Reflexiones desde la Práctica (pp. 177-192). Viña del Mar: Ril Editores.

Canessa, P. \& Guerra, C. (2010). Programa de entrenamiento para nińos victimizados sexualmente que deben declarar en un juicio oral: aportes de la terapia cognitivo conductual. En V. Arredondo y E. Toro (Ed.) Espejos de Infancia: Análisis e Intervenciones en violencia infantil (pp. 89-106). Editado por Corporación de Promoción y Apoyo a la Infancia - Paicabi. 
Celia, M. (2008). ¿El juzgamiento de delitos sexuales empeora la situación de los niños abusados? Recuperado el 29 de octubre del 2010 desde http://www.psicologiacientifica.com/bv/psicologia-337-6-eljuzgamiento-de-delitos-sexuales-empeora-la-situacion-de-los-ninosabusados.html.

Colegio de Psicólogos de Chile (1999). Código de ética profesional. Colegio de Psicólogos de Chile.

Echeburúa, E., Corral, P. \& Amor, P.J. (2002). Evaluación del daño psicológico en las víctimas de delitos violentos. Psicothema, 14 (suplemento), 139-146.

Echeburúa, E. \& Subijana, J. (2008). Guía de buena práctica psicológica en el tratamiento judicial de los niños abusados sexualmente. International Journal of Clinical and Health Psychology, 8(3), 733-749.

Gutiérrez, C., Coronel, E. \& Pérez, C. (2009). Revisión teórica del concepto de victimización secundaria. Liberabit, 15(1), 49-58.

Hernández, J. \& Miranda, M. (2005). ¿Deben declarar los menores victimizados en el acto del juicio oral? Revista La Ley, 6335, 1-5.

Lewis, J. (2003). The mental health of Crime Victims: Impact of legal intervention. Journal of Traumatic Stress, 16(2), 159-166.

Lipovsky, J. (1997). Preparing Children for Court: An interdisciplinary view. Child Maltreat, 2(2), 150-163.

Michels, A. (2009). Psychosocial Support for Children: Protecting the rights of child victims and witnesses in transitional justice processes, Innocenti Working Paper No. 2010-14, Florence, Unicef Innocenti Research Centre.

Plotnikoff, J. \& Woolfson, R. (2001). An evaluation of child witness support. Scottish Executive Central Research Unit. The Scottish Executive Central Research, Edinburgh. Recuperado el 29 de octubre del 2010 desde http://www.sciesocialcareonline.org.uk/repository/ fulltext/cruchildwitnessfull.pdf.

Plotnikoff, J. \& Woolfson, R. (2007). Evaluation of young witness support: examining the impact on witnesses and the criminal justice system. Ministery of Justice, Scotland.

Reno, J., Holder, E., Fisher, R., Robinson, L., Brennan, N. \& Turman, K. (1999). Breaking the Cycle of Violence: Recommendations to Improve the Criminal Justice Response to Child Victims and Witnesses. Office for Victims of Crime, U.S. Deparment of Justice, Washington D.C.

Rubio, M. \& Monteros, S. (2001). Las víctimas de agresiones sexuales ante el sistema jurídico-legal. Anuario de Psicología Jurídica, 11, 59-77.

Seisdedos, N. (1990). STAIC, Cuestionario de autoevaluación. Madrid: TEA Ediciones S.A. 
Unicef (1989). Convención de los derechos del niño. Recuperado el 29 de octubre del 2010 desde http://www.Unicef.es/derechos/docs/ CDN_06.pdf

Unicef (2007). Child protection in Emergencies: priorities, principles and practices. Recuperado el 29 de octubre del 2010 desde http://www. essex.ac.uk/armedcon/story_id/000721.pdf

Fecha de recepción: 29 de octubre de 2010.

Fecha de aceptación: 09 de diciembre de 2011. 
